\title{
Overcoming Difficulties Related with Persistent Left Superior Vena Cava
}

\author{
Serkan Cay ${ }^{1}$, Ozcan Ozeke ${ }^{1}$, Firat Ozcan ${ }^{1}$, Serkan Topaloglu ${ }^{1}$, and Dursun Aras $^{1}$ \\ ${ }^{1}$ University of Health Sciences, Ankara City Hospital
}

May 11, 2020

Letter to the Editor

\section{Overcoming Difficulties Related with Persistent Left Superior Vena Cava}

Serkan Cay, MD; Ozcan Ozeke, MD; Firat Ozcan, MD; Serkan Topaloglu, MD; Dursun Aras, MD

Department of Cardiology, Division of Arrhythmia and Electrophysiology, University of Health

Sciences, Ankara City Hospital, Ankara, Turkey

Correspondence

Serkan Cay, MD

Department of Cardiology,

Division of Arrhythmia and Electrophysiology,

University of Health Sciences, Ankara City Hospital,

Bilkent, 06800 Cankaya, Ankara, Turkey

E-mail: cayserkan@yahoo.com

To the Editor,

We have read with great interest the article entitled 'Cardiac implantable electronic devices in patients with persistent left superior vena cava - A single center experience' by Ghazzal et al ${ }^{1}$ in the latest issue of the journal. We would like to thank the authors for their successful management of patients with persistent left superior vena cava (PLSVC) who consecutively underwent cardiac implantable electronic device implantation. As stated by the authors, various tools and techniques can be used to overcome difficulties when implanting a device through PLSVC. Lead implantation in the right-heart chambers, particularly in the right ventricle, can be challenging due to anatomic relationship between the ostium of the coronary sinus and the right ventricular inflow. Shaping the stylet with 2 or more curves can help to direct the lead tip toward the tricuspid annulus. ${ }^{2}$ Also, with the use of long sheaths such as the standard coronary sinus sheath or steerable electrophysiology sheath, one can cross the valve to achieve the right ventricular lead implantation. Another difficulty is the optimal orientation of the coronary sinus sheath and implantation of the left ventricular lead in the targeted branch of the coronary sinus. Inferior approach from the femoral vein can also be used to cannulate the targeted branch and to implant the electrode. After optimal implantation with good electrical parameters, the proximal end of the electrode can be moved from the groin to the pectoral region using a snare or large bore catheters via PLSVC. Previously described this inferior approach can also be used to implant the right ventricular lead. ${ }^{3,4}$ 
Preprocedural imaging, not only for device implantations but also for electrophysiologic procedures (Figure), is an important step for the detection of PLSVC. ${ }^{5}$ During transthoracic echocardiography, visualization of an enlarged coronary sinus can be related with the presence of a PLSVC. Preprocedural diagnosis of PLSVC can be achieved using contrast imaging. When agitated saline is injected via the superficial veins of the left arm, bubbles appear first in the coronary sinus before the right-heart chambers. For further confirmation of PLSVC and imaging of other anatomic structures including the coronary sinus and its branches, 3D imaging modalities such as computed tomography angiography and magnetic resonance imaging can be used. ${ }^{5}$

Last, previous medical interventional history such as bypass surgery can prove the presence of a PLSVC before the procedure.

Keywords: coronary sinus; imaging; implantation; persistent left superior vena cava

\section{Funding}

None.

\section{Conflict of interests}

The authors declare that there are no conflict of interests.

\section{References}

1 - Ghazzal B, Sabayon D, Kiani S, et al. Cardiac implantable electronic devices in patients with persistent left superior vena cava-A single center experience. J Cardiovasc Electrophysiol . 2020;31(5):1175-1181.

2 - Ozeke O, Akdi A, Cay S, Ozcan F, Aras D, Topaloglu S. Implantation of a single coil ICD via persistent left superior vena cava. Int J Cardiol . 2016;208:26-27.

3 - van Gelder BM, Houthuizen P, Bracke FA. Transseptal left ventricular endocardial pacing: preliminary experience from a femoral approach with subclavian pull-through. Europace . 2011;13(10):1454-1458.

4 - Elencwajg B, López Cabanillas N, Cardinali EL, et al. The Jurdham procedure: endocardial left ventricular lead insertion via a femoral transseptal sheath for cardiac resynchronization therapy pectoral device implantation. Heart Rhythm . 2012;9(11):1798-1804.

5 - Aras D, Cay S, Topaloglu S, Ozcan F, Ozeke O. A rare localization for non-pulmonary vein trigger of atrial fibrillation: persistent left superior vena cava. Int J Cardiol . 2015;187:235-236.

\section{Figure legend}

Figure: 3D computed tomography angiography images of PLSVC in anterior (A) and posterior (B) projections. 3D electroanatomic mapping images of PLSVC (C) and neighboring left-heart structures (D) in the right anterior oblique projection.

CS, coronary sinus; LA, left atrium; LAA, left atrial appendage; LIPV, left inferior pulmonary vein; LSPV, left superior pulmonary vein; PLSVC, persistent left superior vena cava; RIPV, right inferior pulmonary vein; RSPV, right superior pulmonary vein.

Reprinted from reference 5, with permission from Elsevier. 


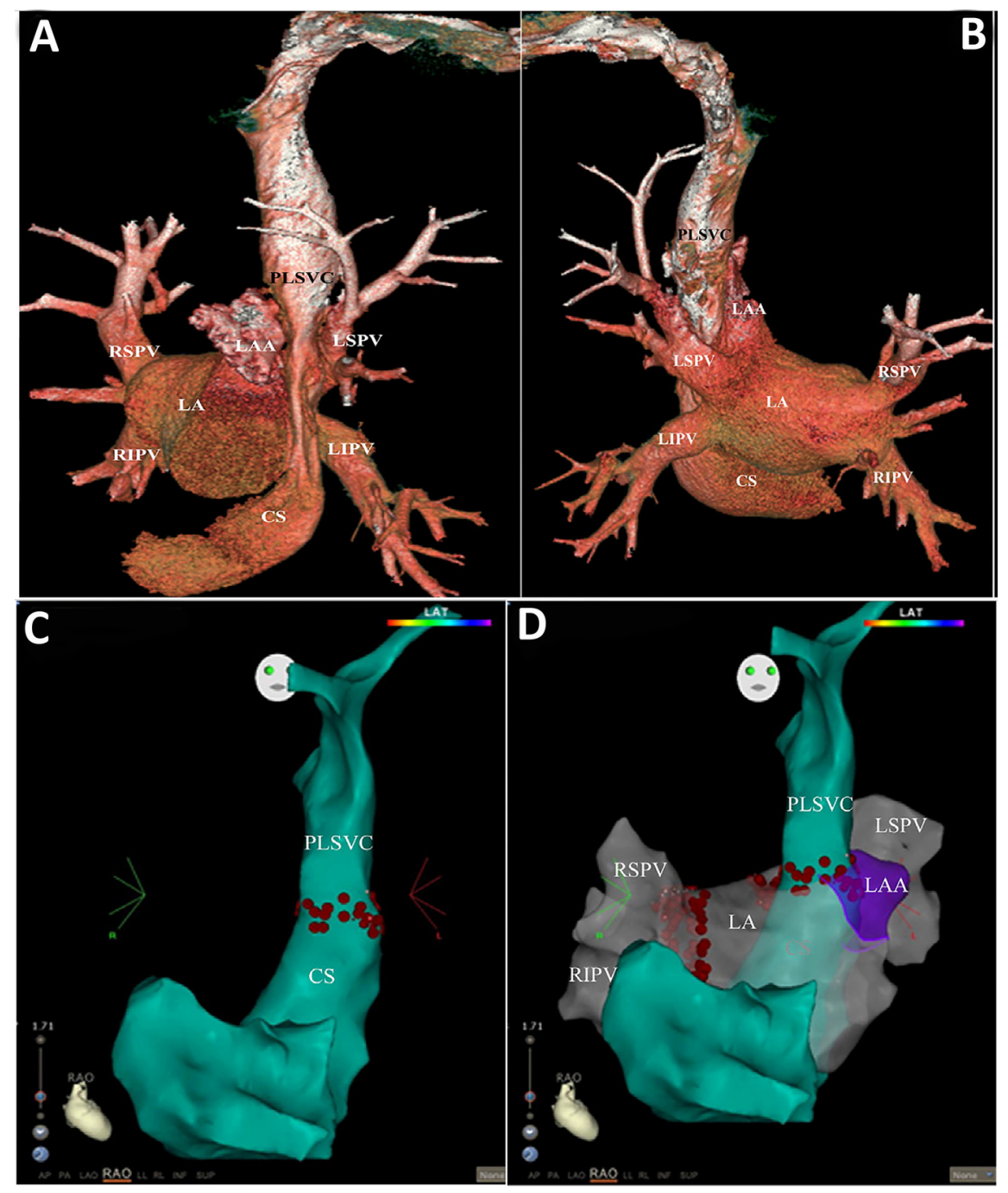

